\title{
LA CANCIÓN DE TIPO POPULAR EN JUAN RAMÓN JIMÉNEZ
}

\author{
Francisco Gutiérrez Carbajo \\ $U N E D$
}

Juan Ramón Jiménez, considerado por algunos críticos como un poeta hermético' y minoritario, se ocupó de la poesía de tipo popular, insertó varias cancioncillas en algunas de sus poemas cultos y cultivó él mismo cantares populares.

Por lo que se refiere al primero de estos aspectos, ya en el prólogo de su Segunda antología poética formula unas observaciones muy precisas sobre lo popular y defiende la sencillez y la espontaneidad como características esenciales de la perfección en el arte. Más tarde, comentando la lírica de Bécquer, explica que este poeta sabe unir «la balada alemana con la poesía popular española» ${ }^{2}$ y sobre las rimas afirmará que constituyen el «son mezclado de la copla popular andaluza y lo nórdico europeo»" ${ }^{3}$. En Crítica paralela observa que lo popular puede ser de dos maneras: o bien lo creado por el poeta anónimo, colectivo, o bien lo que el pueblo acepta de lo elaborado por el poeta tradicional.

Como explica Alvar, «frente al Volkgeist romántico, creador colectivo de la poesía, Juan Ramón cree en la obra del poeta individuo» ${ }^{5}$. La misma postura habia defendido antes Antonio Machado y Álvarez en el Post-scriptum (1883) a los Cantos populares españoles ${ }^{6}$, recogidos por Rodríguez Ma-

' Predmore, M. P.: La poesia hermética de Juan Ramón Jiménez, Madrid, Gredos, 1973.

2 Jiménez, J. R.: El Modernismo. Notas en torno a un curso, México, Aguilar, 1962, p. 175.

${ }^{3}$ Jiménez, J. R.: El trabajo gustoso (conferencias), Selección y prólogo de Francisco Garfias, México, Aguilar, 1961, p. 110.

4 Jiménez, J. R.: Critica paralela, ed. de Arturo del Villar, Madrid, 1975, pp. 202-203.

s Alvar, M.: «Tradicionalidad y popularismo en la teoría literaria de Juan Ramón Jiménez», Cuadernos Hispanoamericanos, CXXV, 376-378 (1981), p. 521.

- Rodriguez Marín, F.: Cantos populares españoles, Sevilla, Francisco Álvarez y Cía., 1882. 
rín, y era, en definitiva la tesis de Milá frente a las teorías románticas del "pueblo-poetà. En esta misma línea se inscriben las formulaciones de Costa, Menéndez Pelayo y Menéndez Pidal.

Juan Ramón no estaba, por lo tanto, al margen de la ciencia filológica del momento, sino que acierta a descubrir, como observa Alvar, algo que a la filología se le había manifestado por otros planteamientos: la tradicionalidad ${ }^{8}$.

Por otra parte, las observaciones de Juan Ramón sobre el tema que nos ocupa presenta una sorprendente analogía con lo que ya en el siglo XVI afirmaba Fray Luis de León: "puede ser que en las ciudades se sepa hablar mejor pero la fineza del sentir es del campo y la soledad»" En términos semejantes se manifiesta el poeta de Moguer en Trabajo gustoso ${ }^{10}$ y en Critica paralela ${ }^{11}$.

Juan Ramón sostiene que el arte y la poesía populares constituyen una imitación de las manifestaciones artificiosas o eruditas: «No hay arte popular, sino imitación, tradición popular del arte» ${ }^{12}$.

Reconoce, sin embargo, que, en el siglo XIX, se produce una inversión de este proceso: la lírica popular alcanza tal auge que los poetas cultos recurren a ella e intentan imitar su código retórico y sus procedimientos expresivos. El fenómeno tampoco es nuevo, ya que, como es sabido, el cultivo de los temas populares y el aprovechamiento de todos los recursos expresivos de la lírica de este tipo constituyen una práctica frecuente en muchos poetas de los siglos XVI y XVII.

Para Juan Ramón, Bécquer y Ferrán son los primeros que llevan a cabo en la poesía española del siglo XIX un acercamiento a la lírica de tipo popular:

\begin{abstract}
«Con Bécquer, con su amigo Augusto Ferrán empieza en la poesía española del siglo XIX, después del presentimiento, la comprensión de lo popular; lo popular en la vida $y$ en el arte, que pronto, en lados diferentes del arte y de la vida, los hombres de la Institución Libre de Enseñanza (...) habian de señalar...."n."
\end{abstract}

${ }^{7}$ Milá y fontanals, M.: Observaciones sobre la poesía popular, Barcelona, Imprenta de Narciso Ramirez, 1853.

"Alvar, M.: op. cit. p. 521.

- Apud. Alvar, op. cit. p. 521.

10 Jiménez, J. R.: El trabajo gustoso... pp. 41-42.

"Jiménez, J. R.: Crítica paralela... pp. 203-204. 262.

12 Jiménez, J. R.: Segunda antologia poética (1898-1918), Madrid, Espasa-Calpe, 1969, p.

i" Jiménez, J. R.: "Dos aspectos de Bécquer», en La corriente infinita, ed. de Francisco Garfias, Madrid, Aguilar, 1961, p. 108. 
Esta preocupación juanramoniana por la poesía de tipo popular no entra en contradicción con el postulado «a la minoría siempre». El propio Juan Ramón, en carta a José Luis Cano aclara que cuando empezó a poner al frente de sus escritos «a la minoría siempre» estaba pensando que esta minoría se encuentra en todas partes, tanto en "el pueblo "cultivado" por sí mismo" como en el «hombre "culturado" en los libros de las ciudades»"

La afición de Juan Ramón a los sones y a los ritmos populares se pone nuevamente de manifiesto en los años finales de la década de los cuarenta: en 1949, en carta a Ángela Figuera confiesa que no le gustan más versos que el octosílabo, «el apropiado de la canción» y el verso libre ${ }^{15}$.

En $E l$ andarin de su órbita declara que aunque le resulte difícil al escritor culto "cojerle el corazón a lo popular», si quiere encontrar lo esencial debe buscarlo en el pueblo, porque éste, que es «humanidad elemental», lo contiene todo como la Naturaleza ${ }^{16}$.

No resulta impertinente, sin embargo, que lo popular reciba siempre en Juan Ramón un tratamiento aristocrático. Esta unión se había fraguado ya, como se explica en Trabajo gustoso, en la Institución Libre de Enseñanza.

El aprovechamiento y la recuperación de lo que hay de aristocrático en lo popular responde a la práctica juanramoniana de la constante selección y depuración poéticas. Por otra parte, en las composiciones de este tipo alcanzan una óptima representación los ideales de sencillez, de sintesis, de justeza, que Juan Ramón solicitaba para su propia lírica.

No parece tampoco censurable, en contra de lo que opina Ángel González ${ }^{17}$, la afirmación citada sobre la «imitación» o «tradición popular del arte». En la génesis de toda obra, popular o culta, hay un genio individual que, firme su producción o no la firme, asume la responsabilidad de ese acto de creación primigenia. En un estadio posterior, la canción que luego será reconocida como un producto de tipo popular, experimenta un proceso de recreación y reelaboración colectivas, el pueblo la canta y la acepta como suya y pasa a ser patrimonio de la comunidad.

Éste es el proceso experimentado, por ejemplo, por muchos cantares de Ruiz Aguilera, de Augusto Ferrán y de otros autores que, según el autor de Espacio, influirán en su propia poesía:

\footnotetext{
14 Apud Alvar, op. cit. p. 524.

is Jiménez, J. R.: Cartas literarias, ed. de Francisco Garfias, Barcelona, Bruguera, col. «Libro Amigo», 1977, p. 175.

${ }_{16}$ Jiménez, J. R.: El andarin de su órbita, selección de Francisco Garfias, Madrid, Magisterio Español, col. Novela y Cuentos, 1974.

1 González, A.: Juan Ramón Jiménez, Madrid, Júcar, 1973, pp. 133-134.
} 
«Este aristócrata popular que fue Augusto Ferrán influye en mi, repito, entre mis quince y mis veinte años, y por unos escasos y breves poemas, tanto como Bécquer, Rosalia de Castro, Jacinto Verdaguer con los suyos, también breves: nunca me cansaré de decirlo»" ${ }^{18}$.

Quizá sean estas influencias, entre otras, las que expliquen que Juan Ramón no se conforme con teorizar sobre la lírica de tipo popular sino que ya muy tempranamente se dedique él mismo a cultivarla.

Uno de los primeros que se refiere a esta dedicación juanramoniana es Rafael Cansinos Assens, que en su Copla andaluza ${ }^{19}$ inserta estas dos soleares compuestas por el poeta de Moguer:

"Me da pena cuando veo
en la alegre primavera
algún arbolillo seco"
"Era el pobrecillo ciego
y cantaba sollozando
la luz de unos ojos negros»

Manuel Alvar ha observado igualmente que Juan Ramón, desde sus primeros libros, da cabida a las creaciones de tipo popular en sus propias composiciones: en Almas de violeta aparecen incrustados unos cantares y las Rimas «se abren con otros de Ferrán por los que siempre sintió especial devoción ${ }^{20}$ :

-Yo no sé lo que tengo, ni sé lo que me hace falta que siempre espero una cosa que no sé cómo se llama.

-Eso que estás esperando dia y noche, y nunca viene; eso que siempre te falta

\footnotetext{
18 Jiménez, J. R.: «Carta a Luis Cernuda», en La corriente infinita, p. 171 (también recogido en Cartas, p. 55).

${ }_{19}$ Cansinos-Assens, R.: La copla andaluza, Madrid, Demófilo, p. 18.

${ }^{20}$ Alvar, M.: op. cit., pp. 522-523.
} 
Aparte de estos cantares de Ferrán, los escritos por Juan Ramón, que reproducen Cansinos Assens y Alvar, pertenecen a una serie de nueve coplas que aparecían ya recogidas en Almas de violeta. De las cancioncillas insertas en Rimas sólo una es realmente nueva; las cuatro restantes se encontraban en el libro anterior.

Pero existían indicios de que Juan Ramón Jiménez había compuesto con anterioridad cantares populares y Graciela Palau se había referido a que el poeta de Moguer los habia publicado en un periódico sevillano. Efectivamente, en el número 18 de «El Programa» de Sevilla aparecen diecisiete cantares escritos por nuestro autor ${ }^{21}$. En esta misma publicación se inserta una crítica a las composiciones juanramonianas firmada por Francisco Ramos Garcia en la que, entre otras cosas, se afirma que en estas canciones «campeaba una nota de candor entre el fuego de una fantasía ardiente, arrebatada, con anhelos de escalar lo increado, para lo que le faltaba aire $)^{22}$. Afirmaciones que no podemos suscribir, ya que estos cantares sí respiran un aire popular y aparecen configurados como las composiciones de este tipo. Enseguida tendremos ocasión de comprobarlo.

Antes de abordar el comentario de las canciones de Juan Ramón nos parece oportuno detenernos en su caracterización del cantar y en el análisis de varios poemas cultos en los que aparecen incrustadas diversas cancioncillas.

Lo que Juan Ramón entendia por copla o canción popular fue expresado poéticamente en varios de sus escritos. En un poema titulado "Canción de canciones", de su libro Piedra y cielo (1917-1918), escribe:

"Canción corta, cancioncilla. Muchas, muchas, muchas... Como estrellas en el cielo, como arenas en la playa, como yerbas en el prado, como ondas en el río. Cancioncilla. Cortas, muchas. Horas, horas, horas, horas. (Estrellas, arenas, yerbas, ondas). Horas, luces; horas, sombras. Horas de las vidas, de las muertes de mi vidan ${ }^{23}$.

${ }^{21}$ El Programa, Sevilla (1899), n. ${ }^{\circ} 18,1$ de junio de 1899.

22 Ramos García, F.: "Galería de escritores y poetas sevillanos: don Juan Ramón Jiménez», El Programa, Sevilla, n. ${ }^{\circ} 21$, del 3 de septiembre, 1899.

${ }^{23}$ Jiménez. J. R.: Piedra y Cielo, en Leyenda, ed. de Sánchez Romeralo, Madrid, CupsaEditorial, 1978, p. 462. 
En la composición número tres de este mismo libro se exalta la emoción y la gracia que emana de la canción:

"¿anción mia,

canta, antes de cantar;

da a quien te mire antes de leerte,

tu emoción y tu gracia;

emánate de ti, fresca y fragante! ${ }^{24}$.

En un poema de La realidad invisible (1917-1924) la canción es identificada con la vida del poeta:

"CANCIÓN; tú eres vida mia, y vivirás, vivirás;

$y$ las bocas que te canten, cantarán eternidad» ${ }^{25}$.

En «Moguer mio", de Pastorales (1901-1905) se prefieren las coplas andaluzas «llenas de azules dorados» a las baladas embrumadas y neblinosas. Los cantos que Juan Ramón confiesa haber encontrado «entre las rosas más bellas del jardín de los románticos ${ }^{26}$ aparecen con frecuencia incrustados en sus composiciones cultas. Así, en el poema "Cádiz» del Diario de un poeta reciencasado (1917) se inserta una cantiña muy popular:

"... Cádiz -igual que un abrazo fino y blanco,

que España, desvelada en nuestra espera,

sacara, en sueños, de su remordimiento

del alba,

todo desnudo sobre el mar dorado-

surje divina.

(Con las bombas que tiran los fanfarrones,

${ }^{24}$ Jiménez, J. R.: Leyenda, ed. citada, p. 449 (con la variante «cantarte» por «leerte»).

${ }^{25}$ Jiménez, J. R.: La realidad invisible, en Leyenda, n. ${ }^{\circ} 846$, p. 472.

26 Jiménez, J. R.: Pastorales con Dios, en Leyenda, n." 188, p. 128. 
se hacen las gaditanas

tirabuzones.)

Los besos matutinos, nuevos

y frescos se levantan

en la brisa toral, a esa blancura...”

En la composición «Los pesares», de Baladas del Monsurio (1907), se utiliza un tango popular como introducción y luego se reproduce varias veces a lo largo del poema:

(Los pesares que tiene tu cuerpo, carne de mi carne, se te vuelvan alegrias)

CANTORA, tú cantabas la tristeza de todos tus dias, el puñal que asesina con sol, la pasión de las sangres sombrías.

(Los pesares que tiene tu cuerpo. carne de mi carne, se te vuelvan alegrias)

La noche estaba mala. Con tu boca tú la enrojecías.

(La guitarra lloraba en tu pecho la tristeza de tus cadadias.)

(Los pesares que tiene tu cuerpo, ${ }^{27}$.

En «Río feliz», también de Baladas del Monsurio, se utiliza una soleá al

${ }^{27}$ Jiménez, J. R.: Baladas del Monsurio, en Leyenda. n. ${ }^{\circ} 234$, pp. 159-60. 
comienzo de la composición como contraste con lo que se expresa a lo largo de todo el poema:

(Aqui no hay nada que ver

porque un barquito que habia

tendió la vela y se fue.)

iAy, que las barcas no quieren ir!

¿Qué hermosa la tarde!

Sus velas blancas no van, están.

¿Qué hermosa la tarde!

¿El río solo limpio de sol!

¿Qué hermosa la tarde!

Las ondas besan, no hay timonel.

¿Qué hermosa la tarde!

¡Ay barcas quietas en agua luz!

¿Qué hermosa la tarde ${ }^{28}$.

Por último, el poema «La madre maltratada», de Domingos del forastero se inicia con esta copla popular:

.. mirala con un cristá
que esa mujé es una madre
por un hijo maltratá
y no la defiende naide ${ }^{29}$.

El préstamo literario es un procedimiento muy frecuente en Juan Ramón, como ha señalado Aurora de Albornoz ${ }^{30}$. Con la incrustación de este tipo de cantos en sus propias composiciones estaba cumpliendo su objetivo de combinar lo popular con lo culto.

Y si Rubén Dario en su crónica La tristeza andaluza escribe que en Arias

28 Jiménez, J. R.: Baladas del Monsurio, en Leyenda, n. ${ }^{\circ}$ 248, p. 172.

29 Jiménez, J. R.: Domingos del forastero, en Leyenda, n. ${ }^{\circ} 450$, p. 298.

30 Albornoz, A. de: «El collage-anuncio en Juan Ramón Jiménez», Revista de Occidente, n. 110 (mayo 1972). 
tristes cantaba la copla andaluza, algunas de las creaciones juanramonianas de su primerá śpoca son auténticos cantares populares. Nos referimos a los publicados en «디 Programa» a los que se aludía más arriba. Muchas ce estals cancicnes van a ser rehechas, $c$ reelaboradas en libros posteriores, siguiendo el criteric de poesia en sucesión o en "transición permanente»". Nos encontramos, en definitiva, ante la concepción de la obra como "poesia abierta» a la quc Juan Ramón se refería frecuentemente.

Los temas que trata Juan Ramón en sus canciones populares son los nucleares de toda su obra lírica: la mujer (amada, amante, el amor), la obra de creación (sujeta casi siempre a un tratamiento metapoético), la muerle, y algún otro tema recurrente como el de los árboles.

Los árboles. cotno cualquier otro elemento de la Naturaleza aparecen en la obra juanramoniana en permanente comunicación con el mundo espiritual del poeta, hasta el punto de que, si en otros creadores puede resultar fácil hablar de la animación como de uno de sus recursos literarios, en el poeta de Moguer aparecen lo animado y lo inanimado en una continua comunicación y. en ocasiones, hasta en una premeditada confusión. Parece como si Juan Ramón siguiese aquel consejo de Novalis que recomendaba la combinación de la Naturaleza con el mundo espritual. Así, si en la primera copla de esta serie el autor se limita a una expresión de su pena ante la visión de un árbol seco en plena primavera, en la segunda se asiste ya a un proceso de comunicación y de diálogo entrañable:
(1) Me da pena cuando veo en la alegre primavera algún arbolillo seco.
(2) ¡Cuán pronto tus flores marchitas cayeron! arbolito que apenas nacía que pronto te has muerto!

En un estadio superior de comunión poética, el árbol aparece incorporado a la propia vida del poeta; así, en el poema «Vuelta», de La realidad invisible se exclamará: "Árbol que traigo en mí, como mi cuerpo, del jardín".

${ }_{24}$ Jiménez, J. R.: Leyenda, ed. citada, p. 449 (con la variante "cantarte» por «leerte»).

La canción (1) le sirve a Cansinos Assens para ilustrar lo que entiende por copla popular y explicar que en tiempos de modernismo y de refinado

"Albornoz, A. de: Prólogo a Nueva antolojía de J. R. J., Barcelona, Peninsula, 1972, p. 26. 
esteticismo, puede florecer aen los planteles de la poesía culta la copla popula- con su sencillez temática $v$ métrica» ${ }^{32}$. Cansinos, siguiendo a Verlaine, define la copla como el poema reducido a sus elementos melódico y emotivo, como el primitivo protoplasma lírico, como el alfa y el omega del poemá.

Las canciones de Juan Ramón se ajustan perfectamente a este esquema diseñado por Cansinos.

Y si más arriba hemos calificado la obra de Juan Ramón como «poesía abierta» o "poesía en transición permanente», este concepto se cumple rigurosamente en el proceso evolutivo que experimenta la copla (1). El cantarcillo vio la luz en el periódico sevillano «El Programa», en su número 18, del 1 de junio de 1899.

Más tarde es incluido, con otras nueve coplas, en la serie "Cantares» del libro Almas de violeta ${ }^{33}$. Con algunas variantes lo volvemos a encontrar en Leyenda ${ }^{34}$. Estas modificaciones afectan al nivel léxico en el que se sustituye "alegre" por "verde»; al orden en la construcción de la frase que invierte la estructura del sintagma («alegre primavera» "primavera verde») $\mathrm{y}$, finalmente, a la configuración métrica que convierte los dos últimos versos de la soleá en un solo verso de dieciséis sílabas:

\author{
Me da pena cuando veo \\ en la alegre primavera algún arbolillo seco.
}

Esta nueva forma que adopta la estrofa -y que se convierte en práctica habitual en Leyenda-, no dificulta el sentido ni entorpece el ritmo de la copla; tampoco impide, por otra parte, el que se reconozca en ella la estructura de la soleá.

Los cambios que experimentan los cantares responden a una labor continua de reflexión que generó una constante hipercorrección de los textos. Así, la sustitución del adjetivo "alegre» por "verde» está motivada seguramente por la obsesión del poeta por el color, que llega a constituir en su obra no un mero elemento decorativo sino un rasgo esencial de su poesía: "Sé que mi obra es lo mismo / que una pintura en el aire», escribió en el poema I de Belleza.

La copla (2), que insiste en el tema de los árboles, no adopta como (1) la forma de la soleá, sino que se configura según el modelo de la seguidilla gitana. En estas estrofas, como es sabido, el verso endecasílabo es sustituido

${ }^{32}$ Cansinos Assens, R.: La copla andaluza, Madrid, Demófilo, 1976, p. 18.

3 Jiménez, J. R.: Almas de violeta, Madrid, Tipografia Moderna, 1900.

${ }^{34}$ Jiménez, J. R.: Leyenda, n. ${ }^{\circ} 31$. 
a veces por un decasílabo o por un dodecasílabo. En la copla que nos ocupa el verso resulta un decasilabo si realizamos una sinalefa en la quinta silaba ("queapenas»), pero si en su lugar efectuamos una pausa medial, como es habitual en la seguidilla gitana, el verso se convierte en un endecasilabo:

\author{
(2) ;Cuán pronto tus flores, // \\ marchitas caveron! // \\ arbolito que / apenas nacia // \\ qué joven te has muerto. ill
}

La copla (2) fue publicada tambièn en el número 18 de «El Programa» y no la hemos visto reproducida en libros posteriores.

Las canciones (1) y (2), aunque con distinta estructura métrica, presentan, sin embargo, afinadades estilisticas. Un elemento esencial en ambas composiciones, desde el punto de vista expresivo, es el diminutivo: este morfema facultativo, con la variedad andaluza -illo ("arbolillo») en (1) y con la modalidad castellana -ito ( "arbolito») en (2), constituye un recurso eficaz de potenciación estilística y de emotividad expresiva, como indica Spitzer. Su papel en la copla andaluza es tan destacado que ha llegado a convertirse, según Amado Alonso, en "una de las convenciones poético-musicales del género» ${ }^{35}$.

Otro grupo de coplas de esta misma serie se centra en el tema de la muerte. La muerte forma parte, para Sánchez Barbudo, del «tema central» de la obra juaramoniana, que se ha definido como «un mismo anhelo: ansia de libertad, salvación de la muerte ${ }^{36}$. Llama la atención en los cantares (3), (4) y (5) el interés tan destacado por la necrofilia, característico de la época romántica, y relativamente frecuente en los escritores modernistas:

\title{
(3) Las tumbas del campo santo parece que están calladas; pero su silencio triste iqué bien lo comprende el alma!
}

(4) Cuando la muerte separa

\footnotetext{
"Alonso, A.: «Noción, emoción, dcción y fantasia en los diminutivos», en Estudios lingüisticos (temas españoles), Madrid. Gredos, 1962, p. 163.

${ }^{3}$ Sánchez Barbudo. A.: La segunda ćpoca de Juan Ramón Jiménez (1916-1953), Madrid, Gredos, 1962, I, p. 11.
} 
1

dos almas que son dichosas

pienso con pena: la muerte

jay! debe ser muy envidiosa.

"Seré siempre tuya"

me dijo en un beso

$y$ entonces sonaron con tristes gemidos

campanas de muerto

Las locuciones "tumbas del camposanto", "triste gemido", "campanas de muerto» revelan una marcada influencia romántica, particularmente de Bécquer. Se patentiza asi en estas composiciones de Juan Ramón la afición por el sentimentalismo tan grato al poeta sevillano. El cementerio está todavía lejos de adquirir el significado simbólico que asume, por ejemplo, en Diario de un poeta reciencasado, y que tan magistralmente ha sido estudiado por Michael P. Predmore ${ }^{37}$.

De estas canciones, si la (4) no volvió a ser publicada después de su inserción en El Programa, no sucedió lo mismo con la (3) y la (5). La (3), después de su primera publicación en 1899, fue incluida en Almas de violeta y más tarde en Leyenda.

Esta reedición de los cantares nos pone de manifiesto que Juan Ramón no se siente atraido por la poesía de tipo popular sólo en su primera época -o en su primer "tiempo", si utilizamos la denominación preferida por nuestro poeta ${ }^{38}$ - sino que muestra el mismo interés a lo largo de toda su trayectoria poética. En el curso de su “obra en sucesión» Juan Ramón va "corrigiendo" y «depurando» los cantarcillos populares y los poemas cultos.

El proceso de "depuración» poética, que consistía básicamente en una eliminación de lo superfluo y en una búsqueda de la palabra justa, transformó la primitiva copla (3) en esta otra:

La tierra del camposanto parece que está callada pero su silencio hondo icómo resuena en el alma. ${ }^{39}$.

En esta nueva reelaboración del cantar, no sólo las tumbas sino toda la

${ }^{37}$ Predmore, M. P.: Op. cit. pp. 46 y ss.

3* Sánchez Barbudo, A.: "Juan Ramón Jiménez: transformación y evolución poética de cuatro temas fundamentales en su obra». Cuadernos Hispanoamericanos, 376-378 (1981), p. 180.

39 Jiménez, J. R.: Leyenda, n." 47. 
tierra aparece impregnada por el silencio. $Y$ este «silencio», por su parte, pierde el calificativo "triste», de resonancias románticas, en favor del adjetivo «hondo», que lo acerca más a lo popular andaluz o al tipo de canción que propugnarían más tarde Manuel Machado y Federico García Lorca.

La imagen del «silencio resonando en el alma» mejora igualmente la del verso de la versión primitiva.

La copla (5) es reproducida también en Almas de violeta. En esta nueva versión se introducen algunas variantes: el tercer verso, quizá para reajustarse más perfectamente a la estructura de la seguidilla gitana, se transforma en endecasilabo; se elimina el adverbio «entonces» y se modifican el tiempo y el aspecto verbales también en el verso tercero. La canción aparece ahora de esta forma:

\author{
"Seré siempre tuya" \\ me dijo en un beso \\ y sonaban con tristes gemidos \\ campanas de muerto...
}

El aspecto durativo de "sonaban" prolonga el tañer de las campanas. A esta misma sensación de morosidad parecen contribuir los puntos suspensivos al final de la estrofa.

En el libro Violeta del naranjal y ninfea del pantano, de Leyenda, la canción ha experimentado nuevas transformaciones: en el primer verso se invierte el orden de la frase; en el segundo, se cambia el "punto de vista" y consiguientemente se modifican las estructuras de los paradigmas pronominal y verbai; los versos tercero y cuarto, por último, son prácticamente nuevos: sólo el «esquilón del muerto» evoca los «tristes gemidos» de las "campanas" de la versión anterior. El sentido de la copla, en Leyenda, se ha vuelto, sin embargo, más hermético:

"iSiempre seré tuya!» le dijo en un verso.

Tú pusiste tu punto final, esquilón del muerto ${ }^{40}$.

La copla (6) se inicia con una expresión de base oral: «el corazón se me

4) Jiménez, J. R.: Levenda, n." 50, p. 31. 
parte». A este grito desgarrador le sucede luego una exlamación más sosegada:

\author{
(6) El corazón se me parte \\ cuando a mi muerta recuerdo; \\ iestá la pobre tan sola, \\ tan sola en el cementerio!
}

Esta copla, después de la primera versión de El Programa, fue incluida en Almas de violeta (1900) y más tarde en Rimas (1902). En Leyenda aparece bastante modificada y la "muerta» es designada con su nombre propio:

\author{
El corazón se me parte \\ si a la Macaria recuerdo \\ ella que fue tan de nadie, \\ tan sola en el rincón seco ${ }^{41}$.
}

La última de las coplas publicadas en El Programa con referencia explícita a la muerte introduce, junto a este tema, el del narcisismo. La combinación de ambos motivos no resulta extraña ya que, como es sabido, el mito de Narciso lleva incorporado el tema de la muerte. asi:

La copla, que en un primer momento puede parecer oscura, se presenta

(7) No comprendo por qué, niña, te causan horror los muertos...; eres joven y eres bella; ¿no te gustan los espejos?...;

No es ésta la primera vez que aparecen asociados, en la obra de Juan Ramón, la muerte, el agua y los espejos; en "La fuente vieja", de Platero $y$ yo se representan asi:

4 Jimenez. J. R.: Leyenda, n." 49, p. 31. 
«Blanca siempre sobre el pinar verde $(. .$.$) encierra tn sí, como una clave o una$ tumba, toda la elegría del mundo, es decir, el sentimiento de la vida verdadera (...). De ella fui a todo. De todo torné a ella. De tal manera está en su sitio. tal armoniosa sencillez la eterniza, el color y la luz son suyos tan por entero, que casi se podría coger de ella en la mano, como su agua, el cuadal completo de la vida (...). Es la cuna y es la boda: es la canción y es el soneto; es la realidad y es la alegria; es la muerte» ${ }^{42}$.

Pero el mito de Narciso conlleva, para Juan Ramón, una fusión con la Naturaleza: Narciso es el hombre que se encuentra con la Naturaleza y quiere confundirse con ella, y en esta fusión busca, precisamente, la superación de la muerte ${ }^{43}$.

Juan Ramón, que se refirió en repetidas ocasiones a los aspectos temáticos y formales de su poesía, declaró que la muerte, la obra y la mujer eran los temas o presencias fundamentales de su obra.

En esta serie de cantares los temas enunciados constituyen los elementos nucleares de las composiciones. Y si en el tratamiento de algunos de ellos, como en el de la muerte, son patentes las resonancias de la lírica becqueriana, este mismo clima romántico envuelve las coplas que tratan del amor. Por otra parte, motivos como los de la «flor marchita y deshojada», "el fuego de los besos», etc., presentan bastantes analogias con los que aparecen en los cantares del sevillano Luis Montoto ${ }^{44}$.

Las canciones (8) y (9), que abordan los temas enumerados, no volvieron a ser reproducidas después de su publicación en El Programa:

(8) Mis besos amantes

tal fuego tenian

que las flores que ha poco me diste

están ya marchitas.

(9) ¿Y tú me preguntas

que por qué está pálida?

¿No sabes que pierde sus frescos matices

la flor deshojada?

42 Jimenez, J. R.: Platero y yo, ed. de R. Gullón. Madrid, Taurus, $1981^{14}$, p. 198.

${ }^{43}$ Gullón. R.: Conversacionés con Juan Rumón, Madrid, Taurus, 1958, p. 121.

${ }_{44}$ Montoto, L.: Melancolia. Sevilla, Rafael Baldaraque, 1872. 
L a influencia de Bécquer es patente en la copla (9) cuyo comienzo nos recuerda la rima XXI del poeta sevillano: “iY tú me lo preguntas?».

L as canciones (8) y (9) construidas segun el modelo de la siguidilla gitana expresan un sentimiento y una melancolía muy lejanos aún del optimismo que más tarde provocarán las flores en nuestro poeta. Así. en "Crepúsculo" del libro Olvidanzas, el poniente invade al poeta "con sus flores de oro". mientras canta el ruiseñor de "todo sus amores». "Las flores huelen a ella", en el poema "Vendaval» del mismo libro. porque "Ayer... fue su cuerpo rosa, / y mio, y rosa, vestido / de seda hlanca, por toda / la casa». El poeta que en "Elejías intermedias", del libro Elejias, llora el «jardín con flores", se lamenta ante la ausencia de las mismas en el poema «Alegres cascabeles», de leyenda $a^{45}$.

En las imágenes de las flores deshojadas y marchitas de estos cantares hay una vaga referencia a la fugacidad de la vida que hinca sus raices en nuestra literatura clásica. Un sentimiento de transitoriedad y de fugacidad provocan también las «estrellas errantes» dt: la canción (10), que tampoco volvió a publicarse después de su inclusion en El Programa:

\author{
(10) Volando en el ciclo, \\ una noche en calmu \\ las azules estreilas erranies \\ iqué pronto se apagan!
}

I as coplas que señalamos con los numerns (11), (12) y (13) se sustentan, desde el punto de vista temático, sobre la dicotomia clerpo/alma. que estructura igualmente otros poemas de esta primera epoca juanramoniana. Esta contraposición entre lo corpóreo y lo sspiritual se resuelve en libros posteriores, como Estio y Belleza.

I a canción (13) fue incluida en Almas de violeta: las dos anteriores no volvieron a ser reproducidas después de su publicación en 1899.

Estas tres canciones, aunque comparten un mismo tema, cada una de ellas se estructura según un modelo estrófico distinto: la (11) se atiene al de la cuarteta asonantada, la (12) al de la soguidilla gitana y la (13) al de la soleá:

\title{
(11) Aunque muy orgullosa seas
}

4s Jiménez. J. R.: Levenda, n.*272, p. 187. 
en orgullo ne me ganas;

tú te precias de tu cuerpo,

yo me precio de tu alma.

(12) ¿Creerás que me importan

tus fieros desdenes?

busca bien en tu oscura conciencia

¡verás cómo pierdes!

(13) Mirad qué arrogante pasa;

icuánto esplendor en su cuerpo!

¡cuánta miseria en su alma!

También fue reproducida en Almas de violeta la siguiente copla:

(14) Farece uria golondrina

su pie no toca la tierra

iay! a algunas criaturas

iqué poco el alma les pesa!

Las canciones (15) y (16) están estructuradas sobre la oposición risa! llanto, y no volvieron a ser publicadas después de su aparición en El Programa:

(15) Hermosa morena.

te adoro yo tanto.

que en llanto convierto, si quieres, mi risa

y en risa mi llanto.

(16) Crees que estriba la ventura

en poder gozar sin tasa

iay! jcuántas veces la risa

hace que broten las lágrimas.

Un nuevo grupo de cantares se centra en el tema de los desheredados $y$ desvalidos: 

(17) Dad siempre a los pobres la limosna Santa; ino es mil veces mejor que el dinero la dicha del alma?
(18) Besad a esos pobres niños que van solos por el mundo sin encontrar pan ni abrigo.
(19) Era el pobrecillo ciego y cantaba sollozando la luz de unos ojos negros.

La copla (17) no volvió a ser reproducida después de su publicación en el Programa; la (18) no apareció en este periódico sino en Rimas y tampoco se publicó más tarde; la (19), sin embargo, experimentó diversas modificaciones, no siempre afortunadas: después de su inclusión en el periódico citado fue publicada en Almas de violeta, en Rimas, y más tarde en Leyenda, donde presenta esta forma:

Era bien ciego aquel ciego

y cantaha sollozando la luz de unos ojos negros.

La ceguera constituye también el tema de la siguiente seguidilla gitana, publicada primero en Almas de violeta y más tarde en Rimas:

(20) ¿Qué divinos eran sus ojos risueños...! ipobrecilla! llorando una pena quedóse sin ellos.

No podia faltar, por último, en los cantares juanramonianos una referencia explícita al último de los grandes temas de su poesía: el de la obra o el de la propia creación literaria. Así, el cantar (21) que fue publicado en El Programa y en Almas de violeta constituye una reflexión sobre el contenido trágico de muchas de estas coplas: 
Se comprueba, así, que Juan Ramón ya en estas creaciones primerizas aborda los temas que más tarde se constituirán en los nucleares de su obra poética. Se pone de manifiesto también, ya en la primera etapa juanramoniana, la práctica de corrección y de depuración de sus textos. Se confirma, además, a tenor de los ejemplos expuestos, el gusto de nuestro autor por las creaciones de tipo popular: métricamente se atiene a los modelos canónicos de la copla andaluza: la cuarteta asonantada o tirana, la seguidilla gitana y la soleá; desde el punto de vista temático, aparte de que la mayoría de los temas tratados son los característicos de las canciones populares, en algunas de ellas, como en (7), irrumpe timidamente el irracionalismo, que constituye, como ha señalado Carlos Bousoño, no sólo una característica de la poesía contemporánea sino también un rasgo singularizador de la de tipo popular. Por último, desde el punto de vista estilístico, se recurre igualmente a ciertos procedimientos de la canción popular andaluza como el diminutivo $(1,2,19,20)$, las frases exclamativas $(2,4,6,10,12,13,16,21)$ y las interrogaciones retóricas $(7,9,12,17)$. 